CHAPTER 14

\title{
A POSSIBILITY OF GENERATION OF SURF BEATS
}

\author{
Sanae Unoki \\ Professor, Faculty of Marine Science and Technology, Tokal University, \\ Shimizu-shi, Shizuoka-ken, Japan \\ Ichiro Isozaki \\ Meteorological Research Institute, \\ Suginami-ku, Tokyo, Japan
}

\section{ABSTRACT}

Indentations of a shoreline or ups and downs of sea bottom form a small oscillating system of water. When the period of natural oscillation in it is near that of advancing sea waves a forced standing oscillation develops there, accompanied by an uneven mean surface level of second order. If the height of the sea waves varies slowly with time, the mean surface fluctuates with the period same as that of the envelope of the sea waves, and such fluctuation will be sent back offshore as a surf beat when it is released by breaking of the original sea waves. The generated long wave seems to be correlated positively or negatively with the envelope of the sea waves, depending on the topography of the basin and the period of the incident sea waves. A possibility of generation of similar long wave in front of a breakwater or a steep beach is also suggested.

\section{INTRODUCTION}

Oscillations of water level whose period is a few minutes are observed on many coasts. Especially they have been familiar among the inhabitants in the vicinity of Omae-zaki in our country with the name of "yappiki" because they made the damage due to storm waves greater once in a while.

Two examples of them, recorded at Hachijo-jima and Omae-zaki in case of severe typhoons, are indicated in (b) and (c) of Fig.I, while (a) presents a conspicuous beat phenomenon of swell with changing wave height. Existence of such long waves was noticed on tidal records by Terada (1912) about fifty years ago, and after that some considerations have been given to the origin of the waves as seen in the following:

Nakano (1939) investigated them in detafl based on tidal records and suggested that they might be caused by long waves generated in a storm. On the other hand, Munk (1949) made a comparison between the records by the tsunami. recorder and the swell recorder, and he inferred in his attractive paper that these oscillations were attributed to the variation of shoreward transports of water by breaking surf with irregular height. He termed them "surf beats".

However, Yoshida (1950) indicated theoretically that variations of water level with a few minutes period could be caused on the open sea on account of non-linear interaction between short waves such as sea or swell, and they could turn to surf beats as they entered the surf zone. Fig. 2 which we owe to him 
(a) Nov 2,1952 (Hochijo-jima)
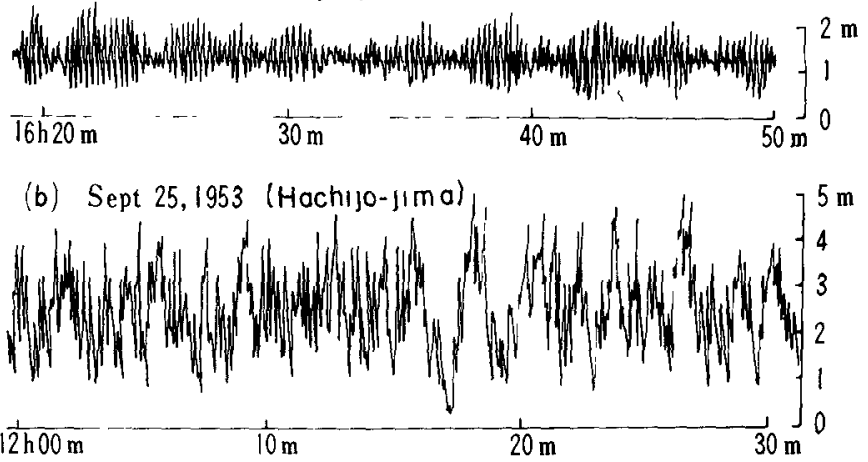

(c) Aug 16,1953

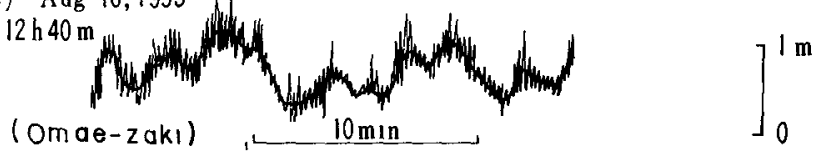

Fig. 1. (a) : A record of swell indicating conspicuous beat phenomenon. (b) and (c) : Examples of long waves with a few minutes period caused by typhoons.

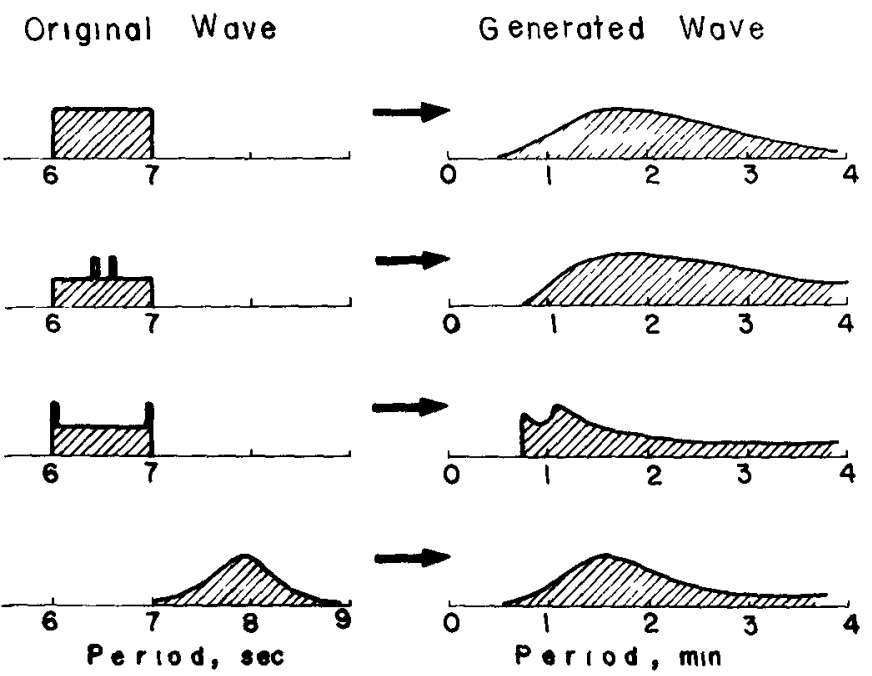

F1g. 2. Assumed shorter-period-spectra and the generated longer-periodspectra, after Yoshida (1950). 
presents spectra of the long waves (right) generated by the assumed spectra of short waves (left). The similar conclusion was independently obtained by Longuet-Higgins and Stewart (1962) on the conception of radiation stress, and they have thrown a light on the explanation of an interesting fact observed by Tucker (1950) that depressions of mean surface level were associated with groups of high waves. This is just inverse to the example observed by Munk (1949), showing positive correlation between the surf beat and the envelope of incoming sea waves.

Recently it was recognized in some of big bays in Japan that the mean sea level inclines and it is higher at the closed end than at the open end in general (Unoki and Isozaki, 1965). From theoretical and numerical considerations such rather conspicuous slope of mean surface could be almost attributed to non-linearity of cooscillating tide in bays. In this report we want to suggest another possibility of generation of surf beats extending this idea to the case of unsteady standing oscillation.

\section{INCLINATION OF MEAN SURFACE LEVEL DUE TO ST ANDING OSCILLATION}

Let us consider a shallow water of one dimension for simplicity. The equations of motion and continuity are

$$
\begin{aligned}
& \frac{\partial u}{\partial t}+u \frac{\partial u}{\partial x}=-g \frac{\partial \zeta}{\partial x}-\frac{x_{w}^{2}}{D} u|u| \\
& \frac{\partial \zeta}{\partial t}=-\frac{\partial}{\partial x}(D u)
\end{aligned}
$$

respectively, where $x$ is a horizontal coordinate taken along the channel, $t$, the time, $u$ the speed of horizontal flow uniform with depth, $\zeta$ the elevation of sea surface, $g$ the acceleration of gravity and $\gamma_{w r}^{2}$ the coefficient of bottcm friction to be assumed constant. $D$ means the momentarily changing depth and it is given by $h+5$ when the depth of the channel is indicated by $h$.

In a basin whose one end is closed, a motion of standing oscillation type occurs and it is expanded as

$$
\begin{aligned}
& s=\bar{\zeta}(x)+\zeta_{I}(x, t)+\zeta_{\mathbb{Z}}(x, t)+\cdots \cdots \\
& u=u_{I}(x, t)+u_{I}(x, t)+\cdots \cdots \cdot \cdots \cdot
\end{aligned}
$$

where $S_{I}$ and $U_{I}$ indicate the standing oscillation of first order and satisfy the linearlized equations, introduced from Eqs, $I$ and $2, \zeta_{I I}$ and $U_{I I}$ that of second order and so on. Though the term $\bar{\xi}$ presenting the mean surface level exists, the term of mean velocity $\bar{u}$ vanishes because the basin is closed at one end and the oscillation is supposed to be steady. (A bar above the variables means an average value with time.) Substituting Eqs. 3 and 4 to Eq. $I$ and neglecting small terms of higher order than the second, we have easily

$$
\frac{\partial}{\partial x}\left(\frac{1}{2} \overline{u_{I}^{2}}\right)=-g \frac{\partial \bar{s}}{\partial x}
$$

after an average with time was taken. By integrating it 


$$
\bar{\zeta}+\frac{1}{2 g} \overline{u_{I}^{2}}=\text { const. }
$$

is obtained. On the other hand, $\overline{\zeta_{I} u_{I}}=0$ is demanded from Eq. 2, but it holds naturaliy in the case of the standing oscillation in question. Eg. 6 tells us that the mean surface level is raised in regions where the current is weak and lowered in regions where the current is strong.

In a case of a basin with a length $l$ and a uniform depth $h$, shown in Fig. 3, such a standing cooscillation as

$$
\begin{aligned}
& \zeta_{1}=a \frac{\cos k(l-x)}{\cos k l} \cos (\sigma t+\varepsilon) \\
& u_{I}=-\frac{a C}{h} \frac{\sin k(l-x)}{\cos k l} \sin (\sigma t+\varepsilon)
\end{aligned}
$$

is caused by the incident wave which is presented

$$
(s)_{x=0}=a \cos (\sigma t+\varepsilon) \text {. }
$$

Here we put $C=\sqrt{g h}$ and $k=\sigma / C$. Assuming $\bar{S}=0$ at the entrance, we have from Egs. 6 and 8

$$
\bar{\zeta}(x)=\frac{a^{2}}{8 h \cos ^{2} k l}\{\cos 2 k(1-x)-\cos 2 k l\}
$$

At the innermost it becomes $\bar{\zeta}(l)=\left(a^{2} / 4 h\right) \tan ^{2} k l$. Similar result has been already obtained by Longuet-Higgins and Stewart (1964) in introducing the radiation stress of standing wave.

According to Fig. 3 we see that the mean surface level becomes high towards the bottom of basin, and in a special case of $k l=\pi / 2$, indicating that the period of extemal wave $(2 \pi / \sigma)$ is equal to that of the fundamental proper oscillation $(4 / / C)$, notable rise of the level is caused because the cooscillation develops remarkably on account of resonance.

Then, suppose that the height of incident waves varies slowly with time. As a matter of fact the mean sea level fluctuates slowly with the period equal to that of the envelope of incident waves. This fluctuation seems to be propagated of $f$ shore out of the basin and maybe freely if the original waves are broken. Such situation is expected on some coasts where ups and doms of sea bottom or indentation of shoreline form a small oscillating system of water whose proper period is near the period of sea or swell, and advancing sea waves with time-changing height may produce surf beats. The problem will be treated theoretically somewhat in the following:

\section{OSCILLATION OF MEAN SURFACE IEVEL CAUSED BY ADVANCING SHORT WAVES WITH TIME-CHANGING HEIGHT}

\footnotetext{
Let's take a model basin shown in Fig.4, in which a small water $A$ with length $l$ and depth $h_{A}$ is found on the coast of a sea $B$ with depth $h_{B}$. We suppose that sea waves are coming with a height changing slowly with time. Basic equations of mean motion of second order, taken over several cycles of tha advancing waves, are
} 

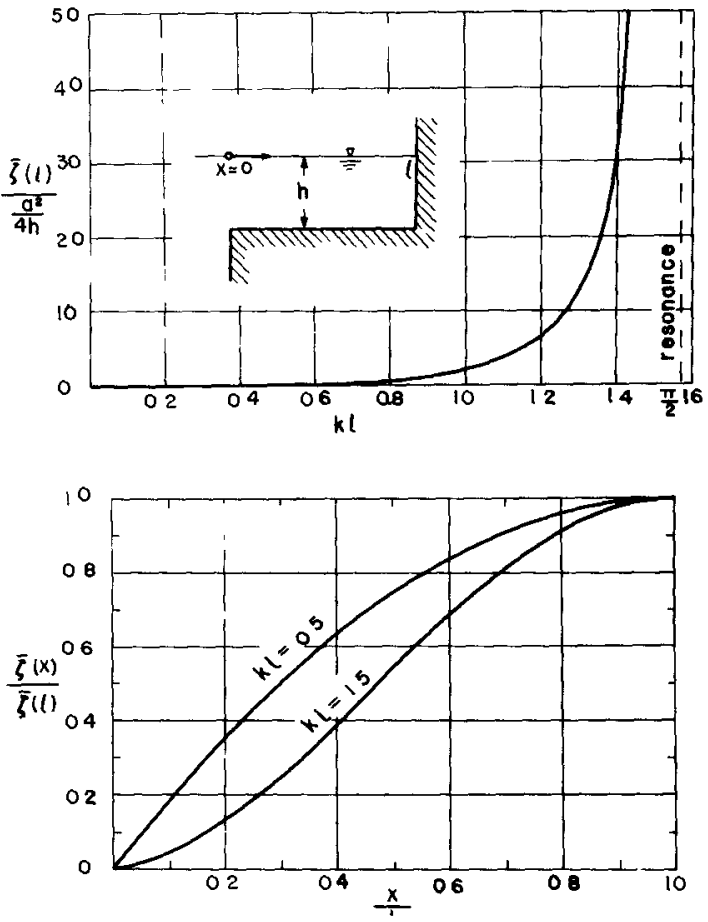

Fig. 3.

Mean surface level associated with the standing cooscillation in a basin of uniform depth. Upper : the level at the innermost, lower : the shape of mean surface.

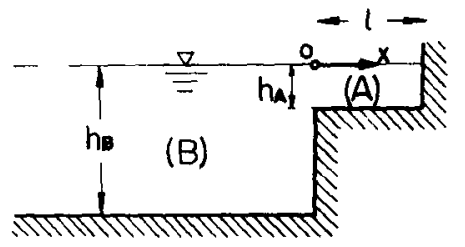

Fig. 4.

A model basin and the amplitude of the fluctuation of mean surface level at the mouth $(x=$ $0)$ and at the innermost $(x=1)$.

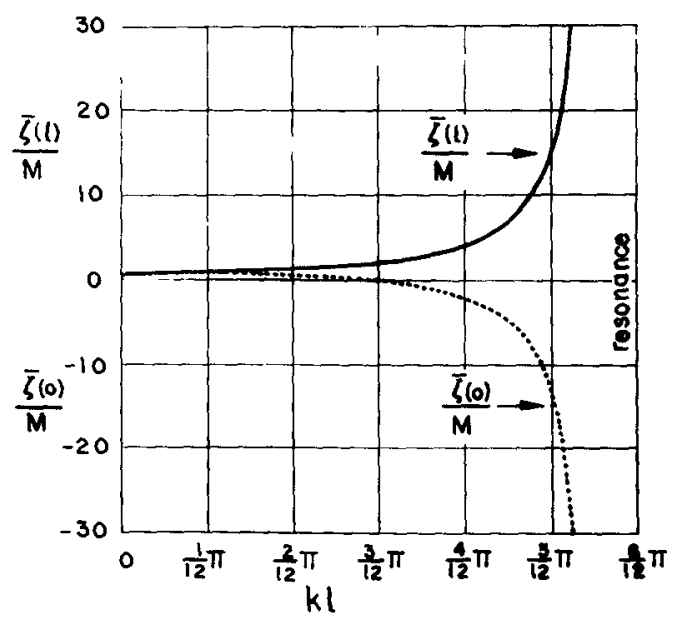




$$
\begin{aligned}
& \frac{\partial \bar{u}_{A}}{\partial t}+g \frac{\partial \bar{S}_{A}}{\partial x}=-\frac{\partial}{\partial x}\left(\frac{1}{2} \overline{u_{I, A}^{2}}\right) \\
& \frac{\partial \bar{S}_{A}}{\partial t}+\frac{\partial}{\partial x}\left(h_{A} \bar{u}_{A}\right)=-\frac{\partial}{\partial x}\left(\overline{S_{I, A} u_{I, A}}\right)
\end{aligned}
$$

for the near-shore water $A$, where $U_{I, A}$ and $\zeta_{I, A}$ indicate the standing cooscillation of first order caused by the incident sea waves. In this case, the mean flow $\bar{u}$ does not vanish since the mean motion is not steady, and the incoming sea waves are regarded as shallow water waves in the water $A$ because of its small depth. On the other hand, for the offshore region $B$ the equations become

$$
\begin{aligned}
& \frac{\partial \bar{u}_{B}}{\partial t}+g \frac{\partial \bar{S}_{B}}{\partial x}=0 \\
& \frac{\partial \bar{S}_{B}}{\partial t}+\frac{\partial}{\partial x}\left(h_{B} \bar{u}_{B}\right)=0
\end{aligned}
$$

on the assumption that the long wave generated in the region $A$ is sent back here. It is also assumed that the water is not necessarily shallow for the original sea waves and non-linearity does not prevail.

Boundary conditions to be satisfied are

$$
\begin{aligned}
& \left(\bar{u}_{A}\right)_{x=1}=0,\left(\bar{\zeta}_{A}\right)_{x=0}=\left(\bar{\zeta}_{B}\right)_{x=0} \\
& b_{A} h_{A}\left(\bar{u}_{A}\right)_{x=0}=b_{B} h_{B}\left(\bar{u}_{B}\right)_{x=0},
\end{aligned}
$$

where $b_{A}$ and $b_{B}$ indicate the breadth of the waters $A$ and $B$ respectively. The advancing sea waves whose amplitude changes slowly are assumed to be

$$
(\zeta)_{x=0}=a(t) \cos (\sigma t+\varepsilon)=H \cos \Delta \sigma t \cos (\sigma t+\varepsilon)
$$

in place of $\mathrm{Eq} .9$.

Since $\Delta \sigma$ is considerably smaller than $\sigma$, it is allowable to presume that the amplitude of the incident waves remains constant for several cycles of them and the cooscillation, $S_{I, A}$ and $U_{I, A}$, is presented by Eqs. 7 and 8 except a constant amplitude $a$ being replaced by $a(t)=H \cos \Delta \sigma t$. Therefore, the fluctuation of mean surface level becomes

$$
\begin{aligned}
& \bar{\zeta}_{A}=M \frac{\cos 2 k_{A}(l-x)}{\cos ^{2} k_{A} l} \cdot \cos 2 \Delta \sigma t \\
& \bar{u}_{A}=-M \frac{\Delta \sigma}{k_{A} h_{A}} \cdot \frac{\sin 2 k_{A}(l-x)}{\cos ^{2} k_{A} l} \cdot \sin 2 \Delta \sigma t
\end{aligned}
$$

for the water $A$, and

$$
\begin{aligned}
& \overline{\zeta_{B}}=M \frac{\left\{\cos ^{2} 2 k_{A} l+\alpha^{2} \sin ^{2} 2 k_{A} l\right\}^{\frac{1}{2}}}{\cos ^{2} k_{A} l} \cdot \cos \left(k_{B} x-\theta\right) \cos 2 \Delta \sigma t \\
& \bar{u}_{B}=M \frac{C_{B}}{h_{B}} \cdot \frac{\left\{\cos ^{2} 2 k_{A} l+\alpha^{2} \sin ^{2} 2 k_{A} l\right\}^{\frac{1}{2}}}{\cos ^{2} k_{A} l} \cdot \sin \left(k_{B} x-\theta\right) \sin 2 \Delta \sigma t(
\end{aligned}
$$


for the sea $B$, where we put

$$
\begin{aligned}
& C_{A}=\sqrt{g h_{A}}, C_{B}=\sqrt{g h_{B}}, k_{A}=\frac{\sigma}{C_{A}}, k_{B}=\frac{2 \Delta \sigma}{C_{B}}, \\
& M=\frac{H^{2}}{16 h_{A}\left\{1-\left(\frac{\Delta \sigma}{\sigma}\right)^{2}\right\}}, \alpha=\frac{b_{A}}{b_{B}}\left(\frac{h_{A}}{h_{B}}\right)^{\frac{1}{2}} \frac{\Delta \sigma}{\sigma}, \tan \theta=\alpha \tan 2 k_{A} l .
\end{aligned}
$$

The level at the mouth of the small basin is

$$
\bar{\zeta}(0) \equiv\left(\bar{\zeta}_{A}\right)_{x=0}=\left(\bar{\zeta}_{B}\right)_{x=0}=M \frac{\cos 2 k_{A} l}{\cos ^{2} k_{A} l} \cos 2 \Delta \sigma t
$$

and one at the inner end

$$
\bar{\zeta}(l) \equiv\left(\bar{\zeta}_{A}\right)_{x=l}=\frac{M}{\cos ^{2} K_{A} l} \cos 2 \Delta \sigma t
$$

From Fig. 4 indicating the amplitude of $\bar{\zeta}(0)$ and $\bar{\zeta}(l)$, we see that the fluctuation of mean sea level is very small and in phase all over the water $A$ in case of $k_{A} l<\pi / 4$. On the contrary, in case of $\pi / 4<k_{A} l<\pi / 2$ the period of natural oscillation approaches that of the incident wave and the amplitude of mean surface level increases greatly, while the phase at the entrance is opposite to that at the innermost. The discussion is to be continued in the same way for the case when $k_{A} l$ exceeds $\pi / 2$.

Variations of the mean surface level as well as the incident waves are illustrated in Fig.5. Comparing (a) with (b) and (c), which belong to the case of $k_{4} l<\pi / 4$, we see that the peak of the mean surface level occurs when groups of high waves arrived at the coast but the height is very small. On the other hand, when the condition of resonance is nearly satisfied, arrival of the high sea waves corresponds to the elevation of the sea level at the innermost (e) but the depression of the sea level at the entrance (d). This rather conspicuous depression of the level is conside red to be freely sent back of fshore as the trough of surf beat when it is released by breaking of the sea waves. This suggests negative correlation between the envelope of the inconing short waves and the generated long waves, corresponding to examples shown by Tucker (1950) though the mechanism of generation may differ.

\section{DISCUSSIONS}

Negative correlation was suggested between the surf beat and the envelope of sea waves in the above section, but a possibility of positive correlation may be considered in such a case as shown below.

In a basin with constant slope of bottom, indicated in Fig.6, regular incident waves with a uniform amplitude produce a standing oscillation given by

$$
\zeta_{1}=a \frac{J_{0}\left\{2^{\frac{1}{2}} k_{*}\left((1-x / l)^{\frac{1}{2}}\right\}\right.}{J_{0}\left(2^{\frac{1}{2}} k_{*} l\right)} \cos (\sigma t+\varepsilon),
$$




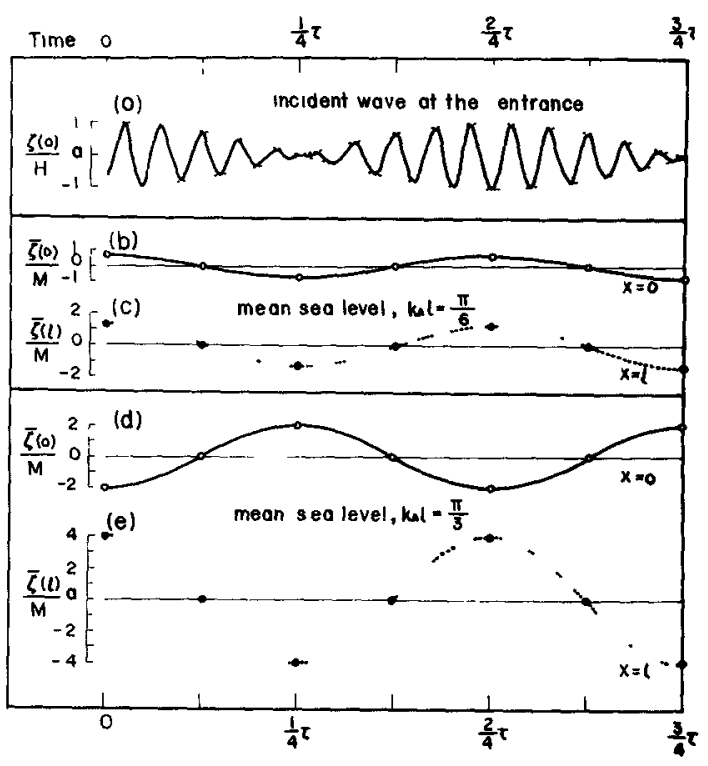

Fig. 5.

(a) : Incident sea waves at the entrance of the near-shore small basin. (b) and (c) : Mean surface level at the entrance $(x=0)$ and at the innermost $(x=1)$ respectively, in case of $k_{A} l=\frac{\pi}{\sigma} \cdot(\mathrm{d})$ and (e) : do, in case of $k_{1} l=\frac{\pi}{3} \cdot \tau \equiv \frac{2 \pi}{4 \sigma}$ means the period of the envelope of incident waves.

Fig. 6.

Mean surface level associated with the standing cooscillation in a basin with constant slope of bottom. Upper : the level at the innermost, lower : the shape of mean surface (cf. Fig. $3)$.
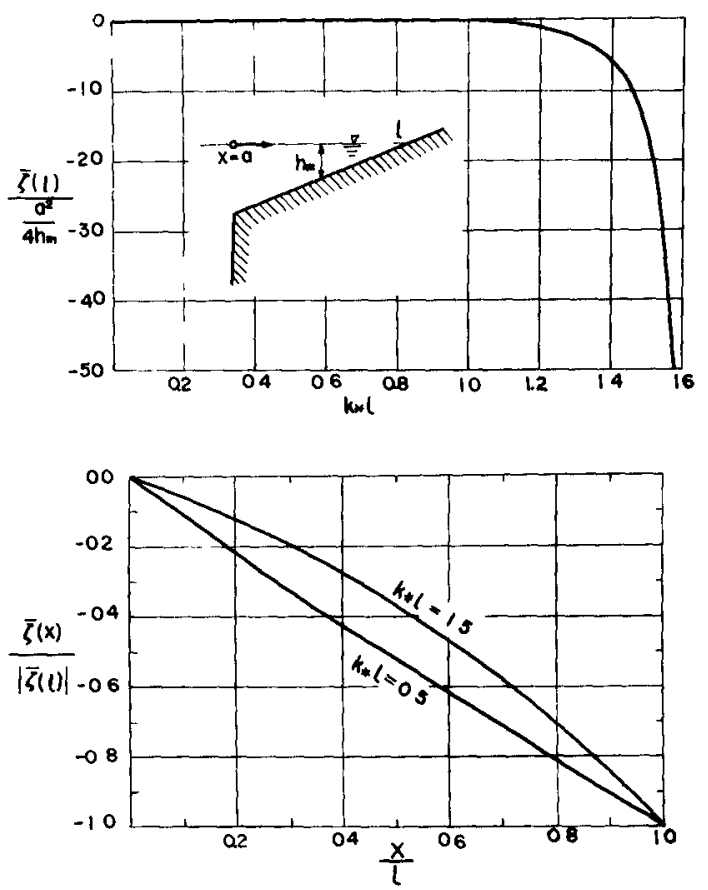


$$
u_{1}=-\frac{a C_{m}}{2^{\frac{1}{2}} h_{m}(1-x / 1)^{\frac{1}{2}}} \cdot \frac{J_{1}\left\{2^{\frac{1}{2}} k_{*} l(1-x / 1)^{\frac{1}{2}}\right\}}{J_{0}\left(2^{\frac{1}{2}} k_{*} l\right)} \sin (\sigma t+\varepsilon)
$$

on the boundary conditions that the sea surface at the entrance fluctuates according to Eq. 9 and the water at the inner end oscillates along the slope of the coast. $J_{0}(\xi)$ and $J_{1}(\xi)$ mean the Bessel functions of zero and first order respectively, and we put $C_{m}=\sqrt{g h_{m}}$ and $k_{*}=\sigma / C_{m}$, where $h_{m}$ presents the mean depth of the basin since the depth is given by $h=2 h_{m}(1-x / l)$.

Substituting Eq. 25 into Eq. 6 and assuming $\overline{5}=0$ at $x=0$, the mean surface level becomes

$$
\bar{\zeta}(x)=\frac{a^{2}}{8 h_{m}} \cdot \frac{\left\{J_{1}\left(2^{\frac{1}{2}} k_{*} l\right)\right\}^{2}-\frac{1}{1-x / 1}\left\{J_{1}\left(2^{\frac{1}{2}} k_{*} /[1-x / 1]^{\frac{1}{3}}\right)\right\}^{2}}{\left\{J_{0}\left(2^{\frac{1}{2}} k_{*} l\right)\right\}^{2}}
$$

At $x=l$ we have

$$
\vec{\zeta}(1)=\frac{a^{2}}{8 h_{m}} \cdot \frac{\left\{J_{1}\left(2^{\frac{1}{2}} k_{*} l\right)\right\}^{2}-\frac{1}{2}\left(k_{*} l\right)^{2}}{\left\{J_{0}\left(2^{\frac{1}{2}} k_{*} l\right)\right\}^{2}} \text {. }
$$

The height of mean surface at the innermost and the shape of it over the basin are indicated in the upper and the lower of Fig.6. The level declines towards the innermost in contrast with the case of uniform depth (Fig.3) and lowering becomes notable when the condition of resonance $\left(k_{*} l \fallingdotseq 1.70\right)$ is satisfied.

The solution in the case when the amplitude of incident waves varies wh time was not obtained. However, taking the result in the case of uniform depth into consideration, it may be expected that the level descends near the innermost but ascends near the entrance when groups of high waves whose period is near that of proper oscillation arrive, and consequently the long wave may be positively correlated with the envelope of incident sea waves. This corresponds to an example observed by Munk (1949) though the mechanism of generation differ. Therefore, surf beats generated through these processes seem to be correlated positively or negatively with the envelope of advancing sea waves, depending on the topography of the small oscillating system of water near the shore and the period of the sea waves.

On the other hand, it is well known that a standing wave or a clapotis is formed in front of a breakwater or a steep beach. So, for the same reason mentioned above, the inclination of mean surface level will be caused by such standing wave, and the mean surface will oscillate if the height of incident sea waves changes slowly with time. Consequently, long waves with the period same as that of the envelope of sea waves may be generated there, too, and be propagated offshore when the incident waves break, more or less.

By the way, the tide presents very distinct beat phenomenon, that is, it is high at the spring tides and low at the neap tides. Accordingly, it is also possible in a bay with conspicuous tide that the sea level fluctuates considerably with a period of around fortnight and it moves out of the bay, suggesting the existence of a new component of long period tides in the neighbouring seas. 


\section{REFERENCES}

Ionguet-Higgins, M.S. and R.W. Stewart (1962): Radiation stress and mass transport in gravity-waves, with application to "surf beats". Jour. Fluid Mech., Vol. 13 , pp. 481-504.

Longuet-Higgins, M.S. and R.W. Stewart (1964): Radiation stresses in water waves, physical discussion, with applications. Deep-Sea Res., Vol. Il, pp. 529-562.

Munk, W.H. (1949): Surf beats. Trans. Amer. Geophys. Union, Vol. 30, pp. 849854 .

Nakano, M. (1939): On the secondary undulations of tides caused by cyclonic storms (In Japanese), Jour. Met. Soc. Japan, Ser. 2, Vol. 17, pp. 140-154; (1949), (In English), Oceanogr. Mag., Vol. 1, pp. 13-32.

Terada, T. (1912): Secondary undulations of tides caused by cyclonic storms. Proc. Tokyo Math. Phys. Soc., and Ser., Vol. 6, pp. 196-201.

Tucker, M.J. (1950): Surf beats; sea waves of 1 to $5 \mathrm{~min}$. period. Proc. Roy. Soc. A, Vol. 202, pp. 565-573.

Unoki. S. and I. Isozaki (1965): Mean sea level in bays, with special reference to the mean slope of sea surface due to the standing oscillation of tide. Oceanogr. Mag., Vol. 17, pp. 11-35.

Yoshida, K. (1950): On the ocean wave spectrum with special reference to the beat phenomena and the "1-3 minute waves". Jour. Oceanogr. Soc., Japan, Vol. 6, pp. 49-56. 